\title{
The Existence and Efficiency of General Equilibrium with Incomplete Markets under Knightian Uncertainty
}

\author{
Wei Ma* \\ Department of Economics, University of Pretoria, South Africa
}

\begin{abstract}
This paper first establishes the existence of equilibrium for an economy with Knightian uncertainty and incomplete markets, and then demonstrates the constrained Pareto efficiency of the equilibrium when there is one commodity only in each state of nature.
\end{abstract}

Keywords: Knightian uncertainty; Incomplete markets; General equilibrium; Pareto efficiency. JEL code: D52; D81

\section{Introduction}

As is well known, Knight (1921) argues that a distinction should be made between risk and uncertainty, and claims that it is uncertainty that prevails in the real world. One way of expressing this distinction is formalized in Bewley (2002) by assuming the existence of a set of probability measures on a state space such that one state-contingent consumption bundle is preferred to another if and only if it has larger expected utility for every measure in that set. According to this formalization, the notion of risk refers to the situation when the set is a singleton, and uncertainty otherwise. Such description of uncertainty is now usually called Knightian uncertainty. It is intuitively obvious that this uncertainty will have an effect on the behavior of economic agents, and it is because of this intuition that Rigotti and Shannon (2005) makes a study of the general equilibrium theory of complete markets under Knightian uncertainty. The purpose of this paper is to continue their analysis by allowing for incomplete markets.

There are several reasons for the market being incomplete, as for instance asymmetric information, moral hazard, and transaction costs (Geanakoplos (1990)). The general equilibrium theory of incomplete markets with standard expected utility (or more generally, with complete preferences) has been extensively studied (Magill and Quinzii (2002)), and a number of interesting results concerning existence of equilibrium and its efficiency have been obtained: for example, Hart (1975) shows that an equilibrium may not exist under standard preference setup, and even when it exists, may not be Pareto efficient.

With this background it appears natural to examine the general equilibrium theory when both Knightian uncertainty and incomplete markets present themselves. The plan of this paper is as follows. Section 2 describes the model, Section 3 establishes the existence of equilibrium, and Section 4 studies its efficiency. Concerning the latter, a general equilibrium with incomplete markets, as said above, is generally speaking not Pareto efficient; but when there is one commodity only in each state of nature, it will be shown in Section 4 to be constrained Pareto efficient. In this section we use, following Magill and Quinzii (2002, Chapter 2, pp. 108-113), a functional-theoretic formalism, which, albeit somewhat abstract, makes the results and their proofs assume a very elegant form. In a sense it is this elegance that justifies the study of the one-commodity model.

\section{The Model}

We study a pure exchange economy with two dates, denoted 0 and 1 , and $S$ possible states of nature at date 1 . We index the states by $s$ running from 1 to $S$, and for notational convenience, call date 0 state 0 . Let there be $m$ consumers, $J$ assets with $J<S$, and $L$ commodities in each state. Suppose that every consumer has $X=R_{+}^{(S+1) L}$ as his consumption

\footnotetext{
${ }^{*}$ Correspondence to: Department of Economics, University of Pretoria, Pretoria 0002, South Africa. Tel: +2712420 4751. Email: maweicityu@gmail.com
} 
space and that consumer $i$ has $\omega^{i} \in X$ as his endowment vector. For $x \in X$, it is often convenient to write it state-wise as $x=\left(x_{0}, \cdots, x_{S}\right)$ with $x_{s} \in R^{L}$.

For preferences, let $>_{i}$ be the preference for consumer $i$, and $\Delta_{S}$ the set of probability measures on $\{1, \ldots, S\}$. We assume that there exists for every consumer $i$ a closed, convex subset $\Pi^{i}$ of $\Delta_{S}$ such that

$$
x^{i}>_{i}\left(x^{i}\right)^{\prime} \text { if, and only if, } U_{\pi}^{i}\left(x^{i}\right)>U_{\pi}^{i}\left(\left(x^{i}\right)^{\prime}\right) \text { for all } \pi \in \Pi^{i},
$$

where $U_{\pi}^{i}\left(x^{i}\right)=u^{i}\left(x_{0}^{i}\right)+\sum_{s=1}^{S} \pi_{s} u^{i}\left(x_{s}^{i}\right)$ and $u^{i}$ is a real-valued, strictly increasing, and concave function on $R_{+}^{L}$. For a behavioral foundation of this representation see Bewley (2002). According to Rigotti and Shannon (2005, p. 237), every $>_{i}$ has an open graph; in what follows we let $P^{i}\left(x^{i}\right)=\left\{\left(x^{i}\right)^{\prime} \in X \mid\left(x^{i}\right)^{\prime}>_{i} x^{i}\right\}$.

We now discuss payoffs of the assets and budget sets of the consumers. Let $q \in R^{J}$ denote a price vector of the assets and $p=\left(p_{0}, \ldots, p_{S}\right) \in R_{+}^{(S+1) L}$ that of the commodities with $p_{s}$ the spot price vector in state $s$. Let $A^{j}=\left(A_{1}^{j}, \cdots, A_{S}^{j}\right) \in$ $R_{+}^{S L}$ be the payoff vector of asset $j$ where $A_{s}^{j} \in R_{+}^{L}$ denotes its promise of the $L$ commodities in state $s$. It is natural to require that for every asset $j$ there exists an $s$ such that $A_{s}^{j} \neq 0$. The nominal return matrix at the price vector $p$ of the assets is then given by

$$
V(p)=\left[\begin{array}{ccc}
p_{1} A_{1}^{1} & \cdots & p_{1} A_{1}^{J} \\
\vdots & & \vdots \\
p_{S} A_{S}^{1} & \cdots & p_{S} A_{S}^{J}
\end{array}\right]=\left[\begin{array}{c}
V_{1}\left(p_{1}\right) \\
\vdots \\
V_{S}\left(p_{S}\right)
\end{array}\right]
$$

Let $\theta^{i} \in R^{J}$ be consumer $i$ 's portfolio, which gives the number of units of each of the $J$ assets he purchases. Then his budget set $B^{i}(p, q)$ is defined to consist of all $\left(x^{i}, \theta^{i}\right)$ satisfying

$$
\begin{aligned}
& p_{0}\left(x_{0}^{i}-\omega_{0}^{i}\right)+q \theta^{i} \leq 0 \\
& p_{s}\left(x_{s}^{i}-\omega_{s}^{i}\right)-V_{s}\left(p_{s}\right) \theta^{i} \leq 0, s=1, \ldots, S .
\end{aligned}
$$

With these preparations we can define the notion of general equilibrium with incomplete markets, henceforth called GEI equilibrium.

Definition 1. A GEI equilibrium is a list $\left(\left(x^{i}, \theta^{i}\right)_{i=1}^{m}, p, q\right)$ satisfying

(I) there exists no $\left(\left(x^{i}\right)^{\prime},\left(\theta^{i}\right)^{\prime}\right) \in B^{i}(p, q)$ such that $\left(x^{i}\right)^{\prime}>_{i} x^{i}$ for $i=1, \ldots, m$;

(II) $\sum_{i}\left(x^{i}-\omega^{i}\right)=0$;

(III) $\sum_{i} \theta^{i}=0$.

\section{Existence of Equilibrium}

It has been shown in Hart (1975) that equilibria may not exist without any restraint on the allowable volume of trade in assets. For this reason and following Radner (1972), we simply place the constraint $\left\|\theta^{i}\right\|_{\infty}<\tau$ for every $i$, where $\tau$ is a positive scalar and $\|\cdot\|_{\infty}$ denotes the $\infty$-norm. Under this condition we can deduce that

Proposition 1. The model of Section 2 has a GEI equilibrium.

Proof. The proof follows the line of argument of Gale and Mas-Colell (1975), and therefore we shall give a brief sketch only and leave the details to the interested reader.

We begin with the introduction of some notation. Let $x^{0}=\sum_{i=1}^{m} x^{i}, \omega^{0}=\sum_{i=1}^{m} \omega^{i}$, and $\theta^{0}=\sum_{i=1}^{m} \theta^{i}$; let $M_{1}=$ $2\left\|\omega^{0}\right\|_{1}, \bar{X}^{i}=\left\{x^{i} \in X \mid\left\|x^{i}\right\|_{\infty} \leq M_{1}\right\}$, and $\Theta^{i}=\left\{\theta^{i} \in R^{J} \mid\left\|\theta^{i}\right\|_{\infty}<\tau\right\}$, where $\|\cdot\|_{1}$ denotes the 1-norm of a vector. Let $V_{0}(p)=\sum_{s=1}^{S} V_{s}\left(p_{s}\right)$; noting that $V_{0}(p)$ is continuous in $p$, we may assume

$$
M_{2}=\max _{\|p\|=1}\left\|V_{0}(p)\right\|_{\infty} .
$$

With this let

$$
\Delta=\left\{(p, q) \in R_{+}^{(S+1) L+J} \mid\|p\|_{1}=1,\|q\|_{\infty} \leq 2 M_{2}\right\} .
$$

Finally let $y^{i}=\left(x^{i}, \theta^{i}\right), y=\left(y^{1}, \ldots, y^{m}\right), \bar{Y}^{i}=\bar{X}^{i} \times \Theta^{i}$, and $\bar{Y}=\prod_{i=1}^{m} \bar{Y}^{i}$. Clearly, $\Delta$ and all $\bar{Y}^{i}$ are convex and compact.

For each $(p, q) \in \Delta$, define

$$
\gamma^{i}(p, q)=\left\{y^{i} \in \bar{Y}^{i} \mid y^{i} \in \operatorname{int}\left(B^{i}(p, q) \cap \bar{Y}^{i}\right)\right\}
$$


where int $(\cdot)$ denotes interior of a set. For the preferences, note that $\pi_{S}$ is possible to vanish for some $\pi=\left(\pi_{1}, \ldots, \pi_{S}\right) \in$ $\Pi^{i}$, and therefore $\gtrsim_{i}$ may not be strongly monotone. To deal with this issue we define a sequence of strongly monotone preferences which converges to $\gtrsim_{i}$. Specifically set

$$
V_{\pi, r}^{i}\left(x^{i}\right)=U_{\pi}^{i}\left(x^{i}\right)+r\left\|x^{i}\right\|_{1},
$$

with $r$ being a positive scalar, and define on $\bar{X}^{i}$ a preference $>_{i, r}$ :

$$
\left(x^{i}\right)^{\prime}>_{i, r} x^{i} \Leftrightarrow V_{\pi, r}^{i}\left(\left(x^{i}\right)^{\prime}\right)>V_{\pi, r}^{i}\left(x^{i}\right) \text { for all } \pi \in \Pi^{i} .
$$

Denote by $E(r)$ the economy with preferences $>_{i, r}$, so that $E(0)$ is our original economy. Define for each $y^{i}=\left(x^{i}, \theta^{i}\right)$

$$
P_{r}^{i}\left(y^{i}\right)=\left\{\left(x^{i}\right)^{\prime} \in \bar{X}^{i} \mid\left(x^{i}\right)^{\prime}>_{i, r} x^{i}\right\} \times \Theta^{i} .
$$

By means of these new preferences we define correspondences $\xi_{r}^{i}: \Delta \times \bar{Y} \rightarrow \bar{Y}_{i}, i=1, \cdots, m$, by

$$
\xi_{r}^{i}(p, q, y)= \begin{cases}\gamma^{i}(p, q), & \text { if } y^{i} \notin B^{i}(p, q), \\ \gamma^{i}(p, q) \cap P_{r}^{i}\left(y^{i}\right), & \text { if } y^{i} \in B^{i}(p, q) ;\end{cases}
$$

and $\xi_{r}^{0}: \Delta \times \bar{Y} \rightarrow \Delta$, by

$$
\xi_{r}^{0}(p, q, y)=\left\{\left(p^{\prime}, q^{\prime}\right) \in \Delta \mid p^{\prime} \cdot\left(x^{0}-\omega^{0}\right)+\left(q^{\prime}-V_{0}\left(p^{\prime}\right)\right) \cdot \theta^{0}>0\right\}
$$

The first step is to show that every $\xi_{r}^{i}, i=0, \ldots, m$, has an open graph. It then follows from the fixed point theorem of Gale and Mas-Colell (1975) that there exists $\left(p_{r}, q_{r}, y_{r}\right) \in \Delta \times \bar{Y}$ satisfying $\xi_{r}^{i}\left(p_{r}, q_{r}, y_{r}\right)=\varnothing, i=0, \ldots, m$.

The second step is to show that $\left(p_{r}, q_{r}, y_{r}\right)$ constitutes an equilibrium for $E(r)$. Using the strong monotonicity of $\gtrsim_{i, r}$ and $\xi_{r}^{i}\left(p_{r}, q_{r}, y_{r}\right)=\varnothing$, this follows from a more or less standard argument. The last step is to show that $\left(p_{r}, q_{r}, y_{r}\right)$ converges to an equilibrium of $E(0)$ as $r \rightarrow 0$; which follows essentially from the fact that every $>_{i}, i=1, \ldots, m$, has an open graph.

\section{Efficiency of Equilibrium}

For the efficiency of a GEI equilibrium, it is not hard to construct an economy with no asset whatsoever whose equilibrium is not Pareto efficient. But, on the other hand, we shall show that a GEI equilibrium is constrained Pareto efficient when there is one commodity only in each state. Let $\operatorname{rint}\left(\Delta_{S}\right)$ denote the relative interior of $\Delta_{S}$; we assume throughout this section that $\Pi^{i} \subset \operatorname{rint}\left(\Delta_{S}\right)$ for every $i$.

When $L=1$, since the budget sets $B^{i}(p, q), i=1, \ldots, m$, are homogeneous of degree zero in prices, we may assume $p=\mathbf{1}$, where $\mathbf{1}=(1, \ldots, 1) \in R^{S+1}$. To simplify the notation let $V=V(\mathbf{1})$ and $B^{i}(q)=B^{i}(\mathbf{1}, q)$. Noting that $V=\left(A_{s}^{j}\right)$, it is without loss of generality to assume $V$ to be of full rank. Let

$$
\bar{V}=\left[\begin{array}{ll}
1 & \\
& V
\end{array}\right]
$$

we define $T: R^{J+1} \rightarrow R^{S+1}$ by

$$
T(\phi)=\bar{V} \phi, \quad \forall \phi \in R^{J+1}
$$

and let $T^{*}: R^{S+1} \rightarrow R^{J+1}$ be the adjoint of $T$. We assume throughout this section that every $u^{i}$ is differentiable and strictly increasing, that is $d u^{i}\left(x_{s}^{i}\right) / d x_{s}^{i}>0$ for all $i$ and all $x_{s}^{i}$. Given $a=\left(a_{1}, \cdots, a_{n}\right)$ with $a_{1} \neq 0$ let $N(a)=$ $\left(1, \frac{a_{2}}{a_{1}}, \cdots, \frac{a_{n}}{a_{1}}\right)$. With this notation we set

$$
\Pi^{i}\left(x^{i}\right)=\left\{N\left(\nabla U_{\pi}^{i}\left(x^{i}\right)\right) \mid \pi \in \Pi^{i}\right\}
$$

where $\nabla U_{\pi}^{i}\left(x^{i}\right)$ is the gradient of $U_{\pi}^{i}\left(x^{i}\right)$ with respect to $x^{i}$. Evidently, this set is compact and convex.

Definition 2. (Magill and Quinzii (2002, Definition 12.1, p. 103)) An allocation $\left(x^{1}, \cdots, x^{m}\right)$ is $V$-feasible if

$$
\text { (i) } \sum_{i}\left(x^{i}-\omega^{i}\right) \leq 0 ; \quad(i i) \text { there exists } \phi^{i} \text { such that } x^{i}-\omega^{i}=T\left(\phi^{i}\right) \text { for every } i
$$

Definition 3. A $V$-feasible allocation $\left(\bar{x}^{1}, \cdots, \bar{x}^{m}\right)$ dominates another $V$-feasible allocation $\left(x^{1}, \cdots, x^{m}\right)$ if $\bar{x}^{i}>_{i} x^{i}$ for all $i .\left(\bar{x}^{1}, \cdots, \bar{x}^{m}\right)$ is constrained Pareto efficient if there exists no $V$-feasible allocation that dominates it. 
The next proposition provides a characterization of constrained Pareto efficiency. It can be thought of as an incomplete-market analogue of Rigotti and Shannon (2005, Theorem 3) and an incomplete-preference analogue of Magill and Quinzii (2002, Corollary 12.6).

Proposition 2. A V-feasible interior allocation $\left(x^{1}, \cdots, x^{m}\right)$ is constrained Pareto efficient if and only if

$$
\bigcap_{i=1}^{m} T^{*}\left(\Pi^{i}\left(x^{i}\right)\right) \neq \varnothing,
$$

where $T^{*}\left(\Pi^{i}\left(x^{i}\right)\right)=\left\{T^{*}(z) \mid z \in \Pi^{i}\left(x^{i}\right)\right\}$.

Proof. (Compare the proof of Theorem 3 of Rigotti and Shannon (2005).) Define

$$
\begin{aligned}
& K_{i}=\left\{\phi^{i} \in R^{J+1} \mid x^{i}+T\left(\phi^{i}\right) \in P_{i}\left(x^{i}\right)\right\}, i=1, \cdots, m, \\
& K_{0}=\sum_{i=1}^{m} K_{i}, \text { and } K=\left\{\phi \in K_{0} \mid \phi=0\right\} .
\end{aligned}
$$

Then $\left(x^{1}, \cdots, x^{m}\right)$ is constrained Pareto efficient if and only if $K=\varnothing$. To prove the proposition, suppose on the one hand that $\bigcap_{i=1}^{m} T^{*}\left(\Pi^{i}\left(x^{i}\right)\right) \neq \varnothing$. Then there exist $z^{i} \in \Pi^{i}\left(x^{i}\right)$ and $q \in R^{J+1}$ such that $T^{*}\left(z^{i}\right)=q$ for all $i$. For any $\phi^{i} \in K_{i}$,

$$
0<U_{\pi}^{i}\left(x^{i}+T\left(\phi^{i}\right)\right)-U_{\pi}^{i}\left(x^{i}\right) \leq \nabla U_{\pi}^{i}\left(x^{i}\right) \cdot T\left(\phi^{i}\right) \forall \pi \in \Pi^{i},
$$

so that $z^{i} \cdot T\left(\phi^{i}\right)>0$. Since $T^{*}$ and $T$ are adjoint, $z^{i} \cdot T\left(\phi^{i}\right)=T^{*}\left(z^{i}\right) \cdot \phi^{i}$, hence $q \cdot \phi^{i}>0$. This implies $q \cdot \phi>0$ for any $\phi \in K_{0}$, hence $K=\varnothing$.

Suppose on the other hand that $K=\varnothing$. Then $0 \notin K_{0}$, so that 0 is not an interior point of $\bar{K}_{0}=\sum_{i=1}^{m}\left(K_{i} \cup\{0\}\right)$. Obviously, $\bar{K}_{0}$ is convex; by the separating hyperplane theorem (cf. Debreu $(1959$, p. 25$\left.)\right)$, there is a $q=\left(q_{1}, \cdots, q_{J+1}\right) \neq$ 0 such that

$$
\inf _{\phi \in \bar{K}_{0}} q \cdot \phi \geq 0
$$

Since $K_{i} \subset \bar{K}_{0}, q \cdot \phi^{i} \geq 0$ for all $\phi^{i} \in K_{i}$. Since $\Pi^{i} \subset \operatorname{rint}\left(\Delta_{S}\right),>_{i}$ is strongly monotone, hence $e_{j}=(0, \cdots, 1, \cdots, 0) \in$ $K_{i}, j=1, \cdots, J+1$. This means $q \geq 0$. We claim $q_{1}>0$. Contrariwise, suppose $q_{1}=0$. Since $e_{1} \in K_{i}$ and $>_{i}$ has an open graph, there exists a neighborhood $\mathscr{O}\left(e_{1}\right)$ of $e_{1}$ with $\mathscr{O}\left(e_{1}\right) \subset K_{i}$. Take $\varepsilon>0$ to be such that $e_{1}^{\prime}=(1,-\varepsilon, \cdots,-\varepsilon) \in \mathscr{O}\left(e_{1}\right)$. As $q_{1}=0, q \geq 0$ and $q \neq 0$, we obtain $q \cdot e_{1}^{\prime}<0$, a contradiction. Hence $q_{1}>0$. Let $\bar{q}=N(q)$; then

$$
\bar{q} \cdot \phi^{i} \geq 0 \quad \text { for all } \phi^{i} \in K_{i}
$$

We claim $\bar{q} \in \bigcap_{i=1}^{m} T^{*}\left(\Pi^{i}\left(x^{i}\right)\right)$. Suppose not; then there is an $i$ such that $\bar{q} \notin T^{*}\left(\Pi^{i}\left(x^{i}\right)\right)$. Since $T^{*}$ is linear and $\Pi^{i}\left(x^{i}\right)$ is compact and convex, so is $T^{*}\left(\Pi^{i}\left(x^{i}\right)\right)$. By the strict separation theorem of convex sets (cf. Magill and Quinzii (2002, p. 73)), there exists a $\phi^{i}=\left(\phi_{1}^{i}, \ldots, \phi_{J+1}^{i}\right)$ satisfying

$$
\bar{q} \cdot \phi^{i}<b<T^{*}\left(z^{i}\right) \cdot \phi^{i}, \quad \text { for all } z^{i} \in \Pi^{i}\left(x^{i}\right) \text { and some } b \in R .
$$

This means $\bar{q} \cdot \phi^{i}<b<z^{i} \cdot T\left(\phi^{i}\right)$ for all $z^{i} \in \Pi^{i}\left(x^{i}\right)$. Take $\bar{\phi}^{i}=\left(\bar{\phi}_{1}^{i}, \ldots, \bar{\phi}_{J+1}^{i}\right)$ with $\bar{\phi}_{1}^{i}=\phi_{1}^{i}-b$ and $\bar{\phi}_{j}^{i}=\phi_{j}^{i}, j=$ $2, \ldots, J+1$; a short calculation shows that

$$
\bar{q} \cdot \bar{\phi}^{i}<0<z^{i} \cdot T\left(\bar{\phi}^{i}\right), \text { for all } z^{i} \in \Pi^{i}\left(x^{i}\right) .
$$

As argued in Rigotti and Shannon (2005, p. 217), we can find an $\alpha>0$ such that $\alpha \bar{\phi}^{i} \in K_{i}$ with $\bar{q} \cdot \alpha \bar{\phi}^{i}<0$. But this contradicts (1).

To proceed further we shall need the following

Lemma 1. For a $V$-feasible allocation $\left(\bar{x}^{1}, \cdots, \bar{x}^{m}\right)$ which is not constrained Pareto efficient, there exists a V-feasible allocation $\left(\tilde{x}^{1}, \cdots, \tilde{x}^{m}\right)$ that dominates it and satisfies $\sum_{i}\left(\tilde{x}^{i}-\omega^{i}\right)=0$. Any V-feasible allocation $\left(\bar{x}^{1}, \cdots, \bar{x}^{m}\right)$ of constrained Pareto efficiency satisfies $\sum_{i}\left(\bar{x}^{i}-\omega^{i}\right)=0$.

Proof. For the first part, let $\left(x^{1}, \cdots, x^{m}\right)$ be any $V$-feasible allocation that dominates $\left(\bar{x}^{1}, \cdots, \bar{x}^{m}\right)$. Let

$$
\tilde{x}^{i}=x^{i}+\frac{1}{m} \sum_{k=1}^{m}\left(\omega^{k}-x^{k}\right) \quad \text { for all } i .
$$


Since $\left(x^{1}, \cdots, x^{m}\right)$ is $V$-feasible, $\sum_{i}\left(x^{i}-\omega^{i}\right) \leq 0$ and $x^{i}-\omega^{i}=T\left(\phi^{i}\right)$ for every $i$ and some $\phi^{i} \in R^{J+1}$. Therefore $\tilde{x}^{i}>_{i} \bar{x}^{i}$. Obviously, $\sum_{i}\left(\tilde{x}^{i}-\omega^{i}\right)=0$. For consumer $i$, we have

$$
\tilde{x}^{i}-\omega^{i}=x^{i}-\omega^{i}+\frac{1}{m} \sum_{k=1}^{m}\left(\omega^{k}-x^{k}\right)=T\left(\tilde{\phi}^{i}\right),
$$

where $\tilde{\phi}^{i}=\phi^{i}-\frac{1}{m} \sum_{k=1}^{m} \phi^{k}$. So $\left(\tilde{x}^{1}, \cdots, \tilde{x}^{m}\right)$ is $V$-feasible. This proves the first part. The second part follows by noting that if $\sum_{i}\left(\bar{x}^{i}-\omega^{i}\right)<0$, there exists a $V$-feasible allocation that dominates $\left(\bar{x}^{i}\right)$.

Now we can state (compare Magill and Quinzii (2002, Theorem 12.3))

Proposition 3. If $\left(\left(\bar{x}^{i}, \bar{\theta}^{i}\right), \bar{q}\right)$ is a GEI equilibrium, then the equilibrium allocation $\left(\bar{x}^{i}\right)$ is constrained Pareto efficient. Conversely, if $\left(\bar{x}^{i}\right)$ is V-feasible and constrained Pareto efficient, there then exist $\left(\bar{\theta}^{i}\right)$ and $\bar{q}$ such that $\left(\left(\bar{x}^{i}, \bar{\theta}^{i}\right), \bar{q}\right)$ is a GEI equilibrium for the economy with endowment structure $\left(\bar{x}^{1}, \cdots, \bar{x}^{m}\right)$.

Proof. For the first part, note that $\left(\bar{x}^{i}\right)$ is $V$-feasible. Suppose on the contrary that $\left(\bar{x}^{i}\right)$ is not constrained Pareto efficient; then there exists, by Lemma 1, an allocation $\left(x^{i}\right)$ and an $\phi^{i}=\left(\phi_{1}^{i}, \theta^{i}\right) \in R^{J+1}$ with $\theta_{i} \in R^{J}$ such that

$$
\text { (i) } x^{i} \in P_{i}\left(\bar{x}^{i}\right) ; \quad \text { (ii) } x^{i}-\bar{x}^{i}=T\left(\phi^{i}\right) ; \quad \text { (iii) } \sum_{i}\left(x^{i}-\bar{x}^{i}\right)=0 .
$$

By (ii), (iii) and the linearity of $T$, we have $\sum_{i} T\left(\phi^{i}\right)=T\left(\sum_{i} \phi^{i}\right)=0$. From the definition of $T$, it follows that $\sum_{i} \phi^{i}=0$. Since $\left(\bar{x}^{i}\right)$ is an equilibrium allocation, we have $x^{i} \notin B^{i}(\bar{q})$. So $x_{0}^{i}-\bar{x}_{0}^{i}+\bar{q} \theta^{i}>0$. Summing over $i$, we obtain

$$
\sum_{i}\left(x_{0}^{i}-\bar{x}_{0}^{i}+\bar{q} \theta^{i}\right)=\sum_{i}\left(x_{0}^{i}-\bar{x}_{0}^{i}\right)>0 .
$$

But this contradicts (iii); so $\left(\bar{x}^{i}\right)$ must be constrained Pareto efficient.

For the second part, if an allocation $\left(\bar{x}^{i}\right)$ is constrained Pareto efficient, there exists, from Proposition 2, a $q$ such that

$$
q \in \bigcap_{i=1}^{m} T^{*}\left(\Pi^{i}\left(\bar{x}^{i}\right)\right) .
$$

Let $q=T^{*}\left(z^{i}\right)$ for some $z^{i} \in \Pi^{i}\left(x^{i}\right), i=1, \ldots, m$, and let $N(q)=(1, \bar{q})$. Then $\left(\left(\bar{x}^{i}, 0\right), \bar{q}\right)$ is a GEI equilibrium of the economy with endowment structure $\left(\bar{x}^{1}, \cdots, \bar{x}^{m}\right)$. To prove this, it suffices to show

$$
P_{i}\left(\bar{x}^{i}\right) \cap B^{i}(\bar{q})=\varnothing .
$$

Suppose not; then there exist an $x^{i} \in P_{i}\left(\bar{x}^{i}\right)$ and a $\theta^{i} \in R^{J}$ such that

$$
x^{i}-\bar{x}^{i}=\left[\begin{array}{c}
-\bar{q} \theta^{i} \\
V \theta^{i}
\end{array}\right] .
$$

Let $\phi^{i}=\left(-\bar{q} \theta^{i}, \theta^{i}\right)$; then $x^{i}-\bar{x}^{i}=T\left(\phi^{i}\right)$. Since $x^{i}>_{i} \bar{x}^{i}$, it follows from the proof of Proposition 2 that $N(q) \cdot \phi^{i}>0$. But a direct calculation yields $N(q) \cdot \phi^{i}=0$. This contradiction shows that (2) must be true.

\section{Acknowledgements}

The author gratefully acknowledges financial support from ERSA(Economic Research Southern Africa).

\section{References}

Bewley, T. F. (2002). Knightian decision theory. part I. Decisions in Economics and Finance, 25(2):79-110.

Debreu, G. (1959). Theory of Value: An Axiomatic Analysis of Economic Equilibrium. Yale University Press.

Gale, D. and Mas-Colell, A. (1975). An equilibrium existence theorem for a general model without ordered preferences. Journal of Mathematical Economics, 2(1):9 - 15.

Geanakoplos, J. (1990). An introduction to general equilibrium with incomplete asset markets. Journal of Mathematical Economics, 19(1-2):1 - 38. 
Hart, O. D. (1975). On the optimality of equilibrium when the market structure is incomplete. Journal of Economic Theory, 11(3):418-443.

Knight, F. H. (1921). Risk, Uncertainty, and Profit. Schaffner \& Marx; Houghton Mifflin Co., Boston, MA: Hart.

Magill, M. and Quinzii, M. (2002). Theory of Incomplete Markets, volume 1. McGraw Hill Book Company.

Radner, R. (1972). Existence of equilibrium of plans, prices, and price expectations in a sequence of markets. Econometrica, 40(2):289-303.

Rigotti, L. and Shannon, C. (2005). Uncertainty and risk in financial markets. Econometrica, 73(1):203-243. 